\title{
The Development of a 3D Body Scan and Composition Database to Assess Body Size Perception in Psychological Research
}

\author{
Nadia MAALIN*1, Sophie M. MOHAMED ${ }^{1}$, Robin S. KRAMER ${ }^{1}$, Andrew IRVINE \\ Piers L. CORNELISSEN² ${ }^{2}$ Kay L. RITCHIE ${ }^{1}$, Martin J. TOVÉE ${ }^{1}$ \\ ${ }^{1}$ School of Psychology, University of Lincoln, Lincoln, England; \\ 2 School of Psychology, Northumbria University, Newcastle Upon Tyne, England
}

https://doi.org/10.15221/19.146

\begin{abstract}
Recently, there has been a shift towards the use of 3D scanning technology in body size perception research. Based on limitations of previous methodologies there is a need to develop and validate female body stimuli that are high-resolution, photo-realistic and biometrically accurate, and modern technology makes this possible. The aim of this project is to generate a database of full-body stimuli using 3D scans of females, varying in both BMI and body composition. This data will be used to generate female body stimuli that can be used in future research to improve the methodological assessment of body image and body size perception in both clinical and non-clinical settings. 3D full-body scans were taken using a 3dMD scanner, body composition data measured using a Tanita bio-impedance scale and physical measurements (chest, waist, hip and arm circumference) taken using a tape measure. The combination of these technologies will enable the statistical mapping of human body shape and composition change and variation across a range of different heights, ages and BMls. The analysis will initially focus on Caucasian females aged 18-45 years old. Data processing techniques and preliminary analysis will be presented and the implications in relation to future research will be discussed.
\end{abstract}

Keywords: body image, 3D body scanning technology, body composition, psychological assessment, body size perception

\section{Introduction}

Body image refers to the "multifaceted psychological experience of embodiment" of one's physical appearance" [1, p.1]. It is a person's mental representation and experience of their own body, including thoughts, feelings and perceptions. Cash and Deagle [2] discuss the historical assessment of body image which has mainly concentrated on two approaches: perceptual and attitudinal/cognitive evaluations of body image. The perceptual approach focuses primarily on the accuracy of body size and shape estimations [3]. This usually involves someone making a visual estimate of their own body size, which is then compared to their actual body size to identify the presence of distorted body image. In order to accurately assess body size and shape estimations, stimulus must capture human body size and shape variation accurately to ensure that valid conclusions can be made.

A variety of methods have been used to assess perceptual body image, each with their own strengths and weaknesses. One well-known method is the use of silhouette body figure scales e.g. 'Stunkard figure rating scale' [4]. This method uses line drawings of human bodies varying in body mass index (BMI) which are not ecologically valid or representative of how we would view bodies in real life. Although, some authors argue that line drawings are beneficial as it ensures that attention is focused purely on body size and shape and not extraneous factors such as, hair, facial features and clothing items [5].

Another commonly used method is photo/video width distortion, where an image of a person is manipulated in the horizontal plane to increase in 'size'. Whilst this method shows good reliability [6], it fails to accurately capture human body size and shape change. Stretching the width of a body will make it appear larger, but it does not depict realistic weight change or body fat storage. Cornelissen et al. [7] state that width distortion is a linear method which creates shape changes that are unrealistic, particularly in the shoulder and hip regions where it can exaggerate the width. 
Overall, both silhouette and width distortion methods lack realism, ecological validity and often fail to accurately capture changes in body shape and composition as body size changes. In order to combat these issues, there has been a shift towards the use of 3D software and computer-generated images (CGIs) when creating stimuli for body image research. The use of these technological advances allows for the maintenance of a high-quality, photo-realistic texture across a range of different body sizes and shapes, and size and shape changes can be based on real-life anthropometric data. Furthermore, stimulus can be created to represent extreme body sizes, which are often hard to capture when using photographs or figural drawings based on real-life people. The use of these this technique improves the ecological and anthropometric validity of the stimulus used in perceptual body image research.

In more recent body image research, there has been a shift towards using 3D scanning technology. 3D scans can be used to create personalised avatars for an individualised assessment of perceptual body image estimations. Personalised avatars can maintain the photo-realistic texture of the individual whilst body size is manipulated [8], and can even maintain some of the individuals' body measurements/proportions (i.e. limb length and height) whilst body size is altered using a statistical mapping of body size variation [9;10]. Furthermore, 3D avatars have been used in both non-clinical and clinical samples e.g. females with Anorexia Nervosa [8] to assess perceptual body image and distortion and may be a useful tool to aid our understanding of body image distortion.

However, the methods discussed above are mostly based on BMI. Whilst BMI is a common and widely used measure of body size, it fails to acknowledge factors such as body fat and muscle mass which could be an important factor when making body size judgements [11]. Some researchers have attempted to rectify this issue by creating figural scales which include changes in body fat and muscle mass rather than solely focusing on BMI $[12,13]$. Nevertheless, like other silhouette figural rating scales, these scales lack realism and ecological validity due to the reliance on using line drawings. Recent research has utilised 3D software to duplicate this scale using CGI images rather than line drawings [14], while this is an improvement in terms of realism, the duplication is based on visual assessments of similarity rather than a statistical, quantitative mapping. Additionally, these body composition scales are developed for use in males, ignoring the role of body compositional factors in female perceptual body image.

Therefore, the aim of this project is to generate a database of 3D, full-body stimuli using 3D scans of females, varying in both body composition and BMI, with the intent of generating body stimuli that can be used in future body image research. This will synthesise the use of high-quality 3D scanning technology with anthropometric data to create stimulus that is appropriately calibrated for both body size and composition. This will help to address the stimulus problems discussed above and aims to improve the measurement of body size perception in psychological and epidemiological research.

\section{Method}

3D full-body scans were taken using a 3dMD 3D body scanner. Participants were asked to stand with their feet shoulder width apart and to raise their arms up to shoulder level with their hands in a fist, to capture a range of arm positions. The 3D scanner provides a full-body 3D mesh of the person and corresponding texture information. Participants were provided with grey underwear to minimise the likelihood of body shape being disguised by clothing and to maintain consistency across the 3D images. Anthropometric measurements were taken from every participant. Full-body and regional body composition was measured using a Tanita bio-impedance analysis (BIA) scale (model MC-780MA). This gives estimates of fat mass, muscle mass, water mass, visceral fat level, weight and BMI. Circumference measurements (in centimetres) were taken from the participants' chest, waist, hips and right arm (relaxed) using a tape measure.

\section{Data analysis}

Overall, data has been collected from 553 individuals in the UK $\left(M_{\text {age }}=34.77, M_{\mathrm{BMI}}=24.60\right)$. The data analysis for this paper will focus on Caucasian females aged between 18 and 45 years old $(n=216$, $M_{\text {age }}=29.19$ and $M_{B M I}=23.89$ ).

Preliminary data analysis is currently on-going for this project and the results will not be reported, however, data processing and statistical analysis techniques are stated below. 


\subsection{Data processing}

The first step of data processing involved using software to ensure that the 3D meshes have a common topology to allow statistical comparisons to be made. For this, scans were processed using Russian 3D Scanner 'Wrap' software [15]. A set of 35 pre-selected landmark points were used by the researchers to match $3 \mathrm{D}$ meshes to a standardised base mesh.

Further data processing was required to reduce the large proportion of variance coming from postural and positional factors, unrelated to body size and shape, which is the primary focus of this analysis. In order to reduce this 'noise', a variety of techniques were used to further prepare the 3D meshes for data analysis. Firstly, heads, hands, and feet were removed as they were not relevant for the analysis. Secondly, the central point of mass was altered so that each limb (torso, left arm, right arm, left arm and left leg) was centred to its centre of mass and then centred to the overall centre of mass. Lastly, a partial Procrustes fit was computed for each limb separately to allow for alignment across the shapes, whilst maintaining individual variability in size and shape. Each finalised mesh consisted of 26,309 vertices.

\subsection{Statistical data analysis of 3D meshes}

A Principal Component Analysis (PCA) will be ran using Matlab 2018a [16] to look at the components which explain the most variance in the $3 \mathrm{D}$ meshes. This will enable the qualitative identification of components related to body size and shape change in this sample of Caucasian females. Correlations between components and body composition variables will be used to identify if there are any relationships between $3 \mathrm{D}$ shape and anthropometric measurements.

\section{Discussion}

Overall, the development of this database allows for the generation of high-quality, 3D full-body stimuli with known anthropometric measurements including BMI, waist-to-hip ratio and body composition, associated with each 3D mesh. Stimulus can be created with the same photorealistic 'identity', ensuring that the focus in on body size and shape without any influence from extraneous variables (see Figure 1). The stimulus can be presented from 360 degrees and can be used as $2 D$ images or life-size and $3 D$ in virtual reality. This database can be used to assess female perceptual body image in clinical and non-clinical samples, with applications beyond perceptual body image research to epidemiological and public health research, for example, creating body scales to investigate perceptions of weight and obesity $[17,18]$. Further analyses are needed to gain a better understanding of the relationship between $3 \mathrm{D}$ body shape and body composition, and to create a quantitative statistical mapping for the creation of full-body 3D stimulus. However, this is the first step in understanding the relationship between body size, shape and body composition in a large sample of female adults for use in future psychological research.
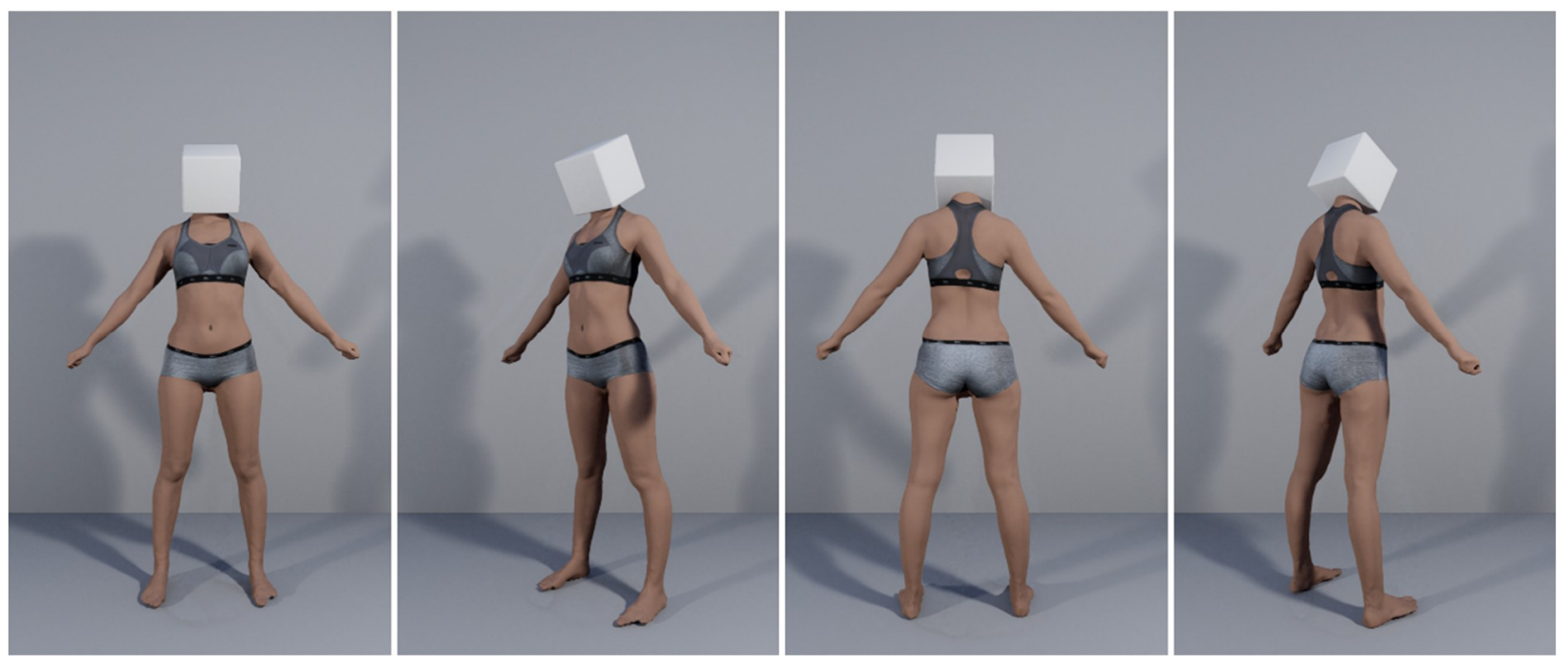

Fig. 1. An example of a female body stimulus, with a computer-generated photo-realistic texture, created using the data collected in this study. 


\section{References}

[1] T. F. Cash, "Body image: Past, present, and future," Body Image, vol. 1, (1), pp. 1-5, 2004.

[2] T. F. Cash and E. A. Deagle, "The nature and extent of body-image disturbances in anorexia nervosa and bulimia nervosa: A meta-analysis," Int. J. Eat. Disord., vol. 22, (2), pp. 107-126, 1997.

[3] R. M. Gardner, "Methodological issues in assessment of the perceptual component of body image disturbance," Br. J. Psychol., vol. 87, (2), pp. 327-337, 1996.

[4] A. J. Stunkard, T. Sorensen and F. Schulsinger, "Use of the Danish Adoption Register for the study of obesity and thinness," Res. Publ. Assoc. Res. Nerv. Ment. Dis., vol. 60, pp. 115-120, 1983.

[5] R. M. Gardner, L. M. Jappe and L. Gardner, "Development and validation of a new figural drawing scale for body-image assessment: the BIAS-BD," J. Clin. Psychol., vol. 65, (1), pp. 113-122, 2009.

[6] M. Probst et al, "The significance of body size estimation in eating disorders: its relationship with clinical and psychological variables," Int. J. Eat. Disord., vol. 24, (2), pp. 167, 1998.

[7] K. K. Cornelissen et al, "The influence of personal BMI on body size estimations and sensitivity to body size change in anorexia spectrum disorders," Body Image, vol. 13, pp. 75-85, 2015.

[8] S. C. Mölbert et al, "Assessing body image in anorexia nervosa using biometric self-avatars in virtual reality: Attitudinal components rather than visual body size estimation are distorted," Psychol. Med., vol. 48, (4), pp. 642-653, 2018.

[9] A. Thaler et al, "Body size estimation of self and others in females varying in BMI," PloS One, vol. 13, (2), pp. e0192152, 2018.

[10] I. V. Piryankova et al, "Can I recognize my body's weight? The influence of shape and texture on the perception of self," ACM Transactions on Applied Perception (TAP), vol. 11, (3), pp. 13, 2014.

[11] V. Groves et al. "How does variation in the body composition of both stimuli and participant modulate self-estimates of men's body size?" Frontiers in Psychiatry, submitted.

[12] G. Cafri and J. K. Thompson, "Evaluating the convergence of muscle appearance attitude measures," Assessment, vol. 11, (3), pp. 224-229, 2004.

[13] H. G. Pope Jr et al, "Body image perception among men in three countries," Am. J. Psychiatry, vol. 157, (8), pp. 1297-1301, 2000.

[14] D. Talbot et al, "Development and validation of the New Somatomorphic Matrix-Male: A figural rating scale for measuring male actual-ideal body discrepancy." Psychology of Men \& Masculinity, 2018.

[15] Russian 3d Scanner, "Wrap 3.3.17", 2018.

[16] The Mathworks Inc., "Matlab 2018a," 2018.

[17] A. Jones et al, "Improving parental recognition of childhood overweight: The map me study," Proc. Nutr. Soc., vol. 72, (OCE4), 2013.

[18] A. Jones et al, "Development of the MapMe intervention body image scales of known weight status for 4-5 and 10-11 year old children," Journal of Public Health, 2017. 